\title{
Hilbert's Fourth Problem: Searching for Harmonic Hyperbolic Worlds of Nature
}

\author{
A. P. Stakhov ${ }^{1,2}$ \\ ${ }^{1}$ International Higher Education Academy of Sciences, Moscow, Russia \\ ${ }^{2}$ Institute of the Golden Section, Academy of Trinitarism, Moscow, Russia \\ Email: goldenmuseum@rogers.com
}

Received June 14, 2013; revised July 15, 2013; accepted September 1, 2013

Copyright (C 2013 A. P. Stakhov. This is an open access article distributed under the Creative Commons Attribution License, which permits unrestricted use, distribution, and reproduction in any medium, provided the original work is properly cited.

\begin{abstract}
Recently the new unique classes of hyperbolic functions-hyperbolic Fibonacci functions based on the "golden ratio", and hyperbolic Fibonacci $\lambda$-functions based on the "metallic proportions" ( $\lambda$ is a given natural number), were introduced in mathematics. The principal distinction of the new classes of hyperbolic functions from the classic hyperbolic functions consists in the fact that they have recursive properties like the Fibonacci numbers (or Fibonacci $\lambda$-numbers), which are "discrete" analogs of these hyperbolic functions. In the classic hyperbolic functions, such relationship with integer numerical sequences does not exist. This unique property of the new hyperbolic functions has been confirmed recently by the new geometric theory of phyllotaxis, created by the Ukrainian researcher Oleg Bodnar ("Bodnar's hyperbolic geometry). These new hyperbolic functions underlie the original solution of Hilbert's Fourth Problem (Alexey Stakhov and Samuil Aranson). These fundamental scientific results are overturning our views on hyperbolic geometry, extending fields of its applications ("Bodnar's hyperbolic geometry") and putting forward the challenge for theoretical natural sciences to search harmonic hyperbolic worlds of Nature. The goal of the present article is to show the uniqueness of these scientific results and their vital importance for theoretical natural sciences and extend the circle of readers. Another objective is to show a deep connection of the new results in hyperbolic geometry with the "harmonic ideas" of Pythagoras, Plato and Euclid.
\end{abstract}

Keywords: hyperbolic Geometry of Lobachevski; Hyperbolic Fibonacci Functions; Bodnar's Geometry of Phyllotaxis; Hilbert's Fourth Problem; New Hyperbolic Worlds of Nature

\section{Introduction}

Recently a number of the important scientific results have been obtained in modern science [1-9]. They relate to hyperbolic geometry and have a direct relation to the theoretical natural sciences in the whole. This direction includes the following scientific results:

1) Fibonacci hyperbolic trigonometry, based on the "golden ratio" [1-3].

2) A new geometric theory of phyllotaxis ("Bodnar's hyperbolic geometry") [4,5].

3) The general theory of hyperbolic functions, based on the "metallic proportions" [6,7].

4) The original solution of Hilbert's Fourth Problem $[7,8]$.

5) The "mathematics of harmony" [9] as a revival of the ancient "mathematics of harmony" (Pythagoras, Plato, Euclid) in modern science.

The purpose of this article is to give a brief survey of these scientific results in order to emphasize their uniqueness and importance for the development of hyperbolic geometry and theoretical natural sciences. The other purpose is to show a deep connection of these results with "harmonic ideas" of Pythagoras, Plato and Euclid. Searching for new hyperbolic worlds of Nature, based on the "golden" and "metallic" proportions, is a reflection of the ancient doctrine on the numerical harmony of Universe in modern mathematics and theoretical natural sciences.

\section{The Uniqueness of the Hyperbolic Fibonacci Functions}

Definitions. Let us compare the classic hyperbolic functions

$$
\operatorname{sh}(x)=\frac{\mathrm{e}^{x}-\mathrm{e}^{-x}}{2} ; \operatorname{ch}(x)=\frac{\mathrm{e}^{x}+\mathrm{e}^{-x}}{2}
$$

with the symmetric hyperbolic Fibonacci functions, in- 
troduced in [2,3]:

Symmetric hyperbolic Fibonacci sine

$$
s F s(x)=\frac{\Phi^{x}-\Phi^{-x}}{\sqrt{5}}
$$

$\underline{\text { Symmetric hyperbolic Fibonacci cosine }}$

$$
c F s(x)=\frac{\Phi^{x}+\Phi^{-x}}{\sqrt{5}}
$$

where $x$ is a continuous variable with values in the range of $\{-\infty \div+\infty\}$

Binet's formulas and the extended Fibonacci numbers. Let us consider the so-called Binet's formulas, introduced by the French mathematician Binet in $19 \mathrm{c}$.:

$$
F_{n}=\left\{\begin{array}{l}
\frac{\Phi^{n}+\Phi^{-n}}{\sqrt{5}} \text { for } n=2 k+1 \\
\frac{\Phi^{n}-\Phi^{-n}}{\sqrt{5}} \text { for } n=2 k .
\end{array}\right.
$$

Binet's formulas (4) define the so-called "extended Fibonacci numbers" (see Table 1).

The extended Fibonacci numbers are an infinite numerical sequence, which is determined in the limits from $-\infty$ to $+\infty$ and has the following unique property:

$$
F_{-n}=(-1)^{n+1} F_{n}
$$

Let us compare Binet's formulas (4) with the symmetric hyperbolic Fibonacci functions (2), (3). This comparison led us to the following result:

$$
F_{n}=\left\{\begin{array}{l}
s F s(n) \text { for } n=2 k \\
c F s(n) \text { for } n=2 k+1
\end{array}\right.
$$

where $k$ takes the values from the set $k=0, \pm 1, \pm 2, \pm 3, \cdots$

This means that for the discrete values of the variable $x(x=0, \pm 1, \pm 2, \pm 3, \cdots)$ the functions (2), (3) coincide with the extended Fibonacci numbers calculated according to Binet's formula (4).

The graphs of the hyperbolic Fibonacci functions. The above unique property of the functions (2), (3) is demonstrated on the graphs of the symmetric hyperbolic Fibonacci functions in Figure 1.

Here the graphs of the hyperbolic sine $y=s F s(x)$ and the hyperbolic cosine $y=c F s(x)$ are represented.

The points on the graph $y=s F s(x)$ correspond to the extended Fibonacci numbers with the even indexes

Table 1. The extended Fibonacci numbers.

\begin{tabular}{cccccccccccc}
\hline$n$ & 0 & 1 & 2 & 3 & 4 & 5 & 6 & 7 & 8 & 9 & 10 \\
$F_{n}$ & 0 & 1 & 1 & 2 & 3 & 4 & 5 & 13 & 21 & 34 & 55 \\
$F_{-n}$ & 0 & 1 & -1 & 2 & -3 & 5 & -8 & 13 & -21 & 34 & -55 \\
\hline
\end{tabular}

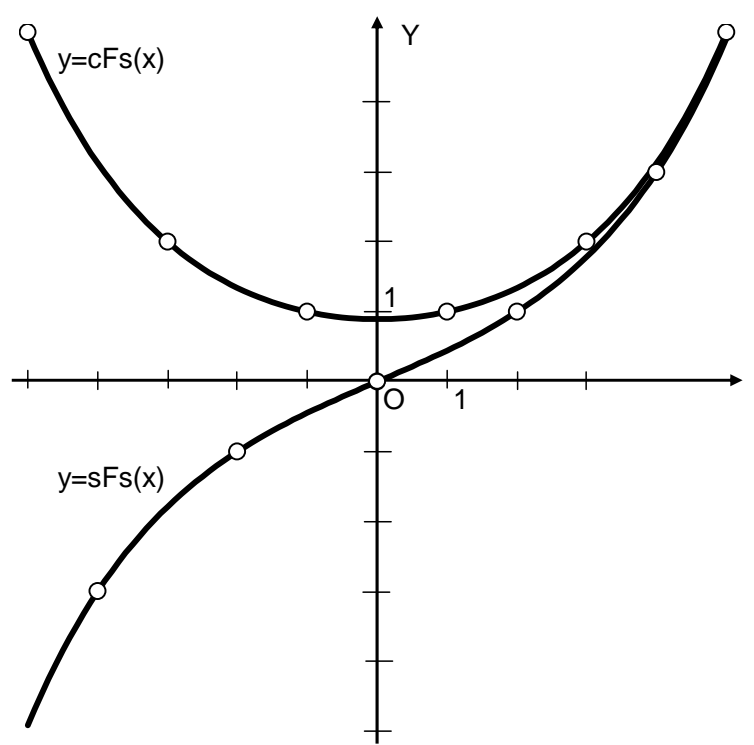

Figure 1. Symmetric hyperbolic Fibonacci functions.

$2 n:$

$$
\begin{aligned}
F_{2 n}= & \left\{\cdots, F_{-8}=-21, F_{-6}=-8, F_{-4}=-3, F_{-2}=-1,\right. \\
& \left.F_{2}=1, F_{4}=3, F_{6}=8, F_{8}=21, \cdots\right\}
\end{aligned}
$$

The points on the graph $y=c F s(x)$ correspond to the extended Fibonacci numbers with the odd indexes $2 n+1$ :

$$
\begin{gathered}
F_{2 n+1}=\left\{\cdots, F_{-7}=13, F_{-5}=5, F_{-3}=2, F_{-1}=1,\right. \\
\left.F_{1}=1, F_{3}=3, F_{5}=5, F_{7}=13, \cdots\right\}
\end{gathered}
$$

Comparison of the hyperbolic Fibonacci functions with the classic hyperbolic functions. It is shown in $[2,3]$, the hyperbolic Fibonacci functions (2), (3) retain all hyperbolic properties of the classic hyperbolic functions (1), however, these hyperbolic functions have two new unique properties. Firstly, the "golden ratio" is the base of these functions and, secondly, they are deeply connected, according to (6), with the extended Fibonacci numbers (4). This means that these functions are, on the one hand, harmonic hyperbolic functions, based on the main harmonic proportion of Nature (the "golden ratio"), and on the other hand, they have the recursive properties, similarly to the extended Fibonacci numbers.

The unique properties $(6-8)$ of the hyperbolic Fibonacci functions (2), (3) are a confirmation of the fact that the hyperbolic Fibonacci functions (2), (3) are a fundamentally new class of hyperbolic functions, which differ from the classic hyperbolic functions (1). The principal distinction of the hyperbolic Fibonacci functions (2), (3) from the classic hyperbolic functions (1) consists in the fact that they own recursive properties like the extended Fibonacci numbers, which are "discrete" analog of the functions (2) and (3) (see Figure 1). In the classic hy- 
perbolic functions (1) such relationship with integer numerical sequences does not exist. This is the uniqueness of the hyperbolic Fibonacci functions (2), (3) in comparison to the classic hyperbolic functions (1). This unique property of the new hyperbolic functions (2), (3) has been confirmed recently by the new geometric theory of phyllotaxis, created by the Ukrainian researcher Oleg Bodnar [4,5].

Taking into account the unique properties of the hyperbolic Fibonacci functions (2), (3), we have every right to say that the hyperbolic geometry, based on the classic hyperbolic functions (1), would have developed differently if the functions (2), (3) were known to Lobachevski.

The authority of Nature (Bodnar's geometry). However, the new geometric theory of phyllotaxis, created by Ukrainian researcher Oleg Bodnar [4,5], is the most powerful confirmation of the uniqueness of the hyperbolic Fibonacci functions. Bodnar has studied growth's problem of the phyllotaxis objects (pine cone, pineapple, cactus, sunflower's head, etc.) and came to the following results.The first key Bodnar's idea is the following: the transformation of the phyllotaxis lattices in the process of its growth are carried out by means of the hyperbolic rotation, the main geometric transformation of hyperbolic geometry [10]. This transformation is accompanied by the modification of the so-called dynamic symmetry [5], which can be simulated by the sequential passage from the object with the smaller symmetry order to the object with the larger symmetry order.

However, the use of the hyperbolic Fibonacci functions (2), (3) for modelling of the growth of phyllotaxis objects is the most important Bodnar's idea. Based on these ideas, Oleg Bodnar came to the special class of hyperbolic geometry, this geometry differs substantially from the classic Lobachevski's geometry or Minkovski's four-dimensional world, based on the classic hyperbolic functions (1). This difference consists in the fact that the main correlations of this geometry are based on the hyperbolic Fibonacci functions (2) and (3), which are connected with the extended Fibonacci numbers by the simple correlation (6).

The most important, that the unique mathematical property (6) is the main cause of the Fibonacci spirals on the surface of the phyllotaxis objects.

The importance of the hyperbolic Fibonacci functions for theoretical natural sciences. "Bodnar's ge- ometry "shows that the "phyllotaxis world "is a hyperbolic world based on the Fibonacci hyperbolic functions (2), (3). These functions are not "fiction" of mathematiccians, they are "natural functions" that are used in natural objects during millions, maybe billions of years long before humanity's appearance. That is why, the hyperbolic Fibonacci functions [2,3], together with "Bodnar's geometry" $[4,5]$ can be attributed to the category of fundamental scientific discoveries, and as such, they are destined to remain in science. The vital importance of the hyperbolic Fibonacci functions (2), (3) for theoretical physics and theoretical natural sciences in the whole follows from these reasoning's.

\section{The Uniqueness of the Hyperbolic Fibonacci $\lambda$-Functions}

Definition. In the works [6,7] the hyperbolic Fibonacci $\lambda$-functions have been introduced:

Hyperbolic Fibonacci $\lambda$-sine

$$
s F_{\lambda}(x)=\frac{\Phi_{\lambda}^{x}-\Phi_{\lambda}^{-x}}{\sqrt{4+\lambda^{2}}}
$$

Hyperbolic Fibonacci $\lambda$-cosine

$$
c F_{\lambda}(x)=\frac{\Phi_{\lambda}^{x}+\Phi_{\lambda}^{-x}}{\sqrt{4+\lambda^{2}}}
$$

where $\lambda=1,2,3, \cdots$ is a given natural number, $\Phi_{\lambda}$ is a positive root of the following algebraic equation:

$$
x^{2}-\lambda x-1=0
$$

The root $\Phi_{\lambda}$ has the following form:

$$
\Phi_{\lambda}=\frac{\lambda+\sqrt{4+\lambda^{2}}}{2}
$$

Note that for the case $\lambda=1$ the formula (13) is reduced to the classic "golden ratio" $\Phi_{1}=\frac{1+\sqrt{5}}{2}$. This means that the formula (13) gives an infinite number of new mathematical constants called "metallic means" or "metallic proportions" by the Argentinean mathematiccian Vera de Spinadel [11].

If we take in (13) $\lambda=1,2,3,4$, then we get the following mathematical constants, having the following names (according to Vera de Spinadel [11]):

It is clear that the number of the "metallic proportions"

$$
\begin{aligned}
& \Phi_{1}=\frac{1+\sqrt{5}}{2}(\text { golden proportion, } \lambda=1) ; \Phi_{2}=1+\sqrt{2}(\text { silver proportion, } \lambda=2) ; \\
& \Phi_{3}=\frac{3+\sqrt{13}}{2}(\text { bronze proportion, } \lambda=3) ; \Phi_{4}=2+\sqrt{5}(\text { cooper proportion, } \lambda=4) .
\end{aligned}
$$

(13) is theoretically infinite. The most significant is the

fact that for the case $\lambda=1$ the formula (13) is reduced 
to the classic "golden ratio". This means that the "metallic proportions" like the "golden ratio" may express some new kinds of "mathematical harmony," which can actually exist in Nature.

Gazale's formulas. The algebraic Equation (12) follows from the recursive relation:

$$
F_{\lambda}(n+2)=\lambda F_{\lambda}(n+1)+F_{\lambda}(n) ; F_{\lambda}(0)=0, F_{\lambda}(1)=1 .
$$

The recursive relation (14) "generates" an infinite number of the new numerical sequences, because every number $\lambda$ "generates" its own numerical sequence (14). The numerical sequences, generated by the recursive relation (14), are called Fibonacci $\lambda$-numbers. For the case $\lambda=1$ we get the classic Fibonacci numbers:

$0,1,1,2,3,5,8,13,21, \ldots$ and for the case $\lambda=2$ we get Pell numbers: $0,1,2,5,12,29,70, \ldots[12]$.

The Fibonacci $\lambda$-numbers have many remarkable properties, similar to the properties of the classic Fibonacci numbers. It is proved that the Fibonacci $\lambda$-numbers, as well as the classical Fibonacci numbers, can be "extended" to the negative values of the discrete variable $n$.

Table 2 shows the four "extended" Fibonacci $\lambda$-sequences, corresponding to the values $\lambda=1,2,3,4$.

It is easy to prove [7] the following generalized Cassini formula for the Fibonacci $\lambda$-numbers:

$$
F_{\lambda}^{2}(n)-F_{\lambda}(n-1) F_{\lambda}(n+1)=(-1)^{n+1}
$$

The formula (14) give the Fibonacci $\lambda$-numbers recursively. However, the Fibonacci $\lambda$-numbers can be represented in analytical form through the "metallic proportions" (13). Such formulas have been deduced by the French mathematician Midhat Gazale [13]:

$$
F_{\lambda}(n)=\left\{\begin{array}{l}
\frac{\Phi_{\lambda}^{n}+\Phi_{\lambda}^{-n}}{\sqrt{4+\lambda^{2}}} \text { for } n=2 k+1 ; \\
\frac{\Phi_{\lambda}^{n}-\Phi_{\lambda}^{-n}}{\sqrt{4+\lambda^{2}}} \text { for } n=2 k
\end{array}\right.
$$

where $k=0, \pm 1, \pm 2, \pm 3, \cdots$.

Gazale's formulas (16) are a generalization of Binet's formulas (4). A distinctive feature of (16) is that they generate an infinite number of the formulas (16), because each natural number $\lambda=1,2,3, \cdots$ corresponds to the different Gazale's formulas (16). In particular, for the case $\lambda=1$ Gazale's formulas (16) are reduced to Binet's formulas (4).

Connection of the hyperbolic Fibonacci $\lambda$-functions with Gazale's formulas. It is proved $[6,7]$ that the hyperbolic Fibonacci $\lambda$-functions (9), (10) coincide with the extended Fibonacci $\lambda$-numbers, given by Gazale's formulas (16) for the discrete values of the variable
Table 2. The "extended" Fibonacci $\lambda$-numbers $(\lambda=$ 1,2,3,4).

\begin{tabular}{cccccccccc}
\hline$n$ & 0 & 1 & 2 & 3 & 4 & 5 & 6 & 7 & 8 \\
$F_{1}(n)$ & 0 & 1 & 1 & 2 & 3 & 4 & 8 & 13 & 21 \\
$F_{1}(-n)$ & 0 & 1 & -1 & 2 & 3 & 5 & 8 & 13 & -21 \\
$F_{1}(n)$ & 0 & 1 & 2 & 5 & 12 & 29 & 70 & 169 & 408 \\
$F_{2}(-n)$ & 0 & 1 & -2 & 5 & -12 & 29 & -70 & 169 & -408 \\
$F_{1}(n)$ & 0 & 1 & 3 & 10 & 33 & 109 & 360 & 1189 & 3927 \\
$F_{3}(-n)$ & 0 & 1 & -3 & 10 & -33 & 109 & 360 & 1189 & 3927 \\
$F_{1}(n)$ & 0 & 1 & 4 & 17 & 72 & 305 & 1292 & 5473 & 23184 \\
$F_{4}(-n)$ & 0 & 1 & -4 & 17 & -72 & 305 & -1292 & 5473 & -23184 \\
\hline
\end{tabular}

$x=n=0, \pm 1, \pm 2, \pm 3, \cdots$, that is,

$$
F_{\lambda}(n)= \begin{cases}s F_{\lambda}(n), & n=2 k \\ c F_{\lambda}(n), & n=2 k+1\end{cases}
$$

The formula (17) determines the uniqueness of the hyperbolic Fibonacci $\lambda$-functions. They retain all the above unique mathematical properties of the symmetric hyperbolic Fibonacci functions (2), (3). A peculiarity of the hyperbolic Fibonacci $\lambda$-functions (9), (10) consists in the fact that a number of the hyperbolic functions, given by (9), (10), is theoretically infinite because every natural number $\lambda=1,2,3, \cdots$ "generates" its own variant of the hyperbolic functions of the kind (9), (10).

\section{A New Approach to Hilbert's Fourth Problem}

Hilbert's Fourth Problem. In the articles [7,8] the original solution of Hilbert's Fourth Problem is described. Hilbert's Fourth Problem [14,15], which relates to the non-Euclidean geometry, was formulated by David Hilbert as follows [16]:

"The more general question now arises: Whether from other suggestive standpoints geometries may not be devised which, with equal right, stand next to Euclidean geometry".

Hilbert's citation contains the formulation of a very important scientific problem, which is of fundamental interest not only for mathematics, but also for all theoretical natural sciences: are there non-Euclidean geometries, which are close to the Euclidean geometry and are interesting from the "other suggestive standpoints?" If we consider it in the context of theoretical natural sciences, then Hilbert's Fourth Problem is about finding NEW HYPERBOLIC WORLDS OF NATURE, which are close to the Euclidean geometry and reflect some new properties of Nature's structures and phenomena.

Unfortunately, the attempts at solving Hilbert's Fourth 
Problem, made by German mathematician Herbert Hamel (1901) and later by the Soviet mathematician Alexey Pogorelov [17] (1974), have not lead to signifycant progress, as described in Wikipedia's articles "Hilbert problems" [14] and the "Fourth Hilbert problem" [15]. In these articles, the status of Hilbert's Fourth Problem is formulated as "too vague to be stated resolved or not" and Pogorelov's solution [17] even is not mentioned in $[14,15]$. About the same point of view on Hilbert's Fourth Problem is presented in the remarkable book [18]. So from the standpoint of Wikipedia's mathematical community, the reason why Hilbert's Fourth Problem is not resolved until now, is own fault of Hilbert, who formulated this problem not clearly enough.

From the "game of postulates" to the game of functions”. According to [19], the reason lies elsewhere. All the known attempts to solve this problem (Hamel, Pogorelov) were in the traditional framework; let's call this the "game of postulates". This "game" started from the works by Gauss, Lobachevski and Bolyai, when Euclid's 5 th postulate was replaced by the opposite one. This was the most major step in the development of the nonEuclidean geometry, called "Lobachevski's geometry". The first non-Euclidean geometry (Lobachevski's geometry), which changed the traditional geometric ideas, is also known as "hyperbolic geometry". This name highlights the fact that this geometry is based on the hyperbolic functions (1).

It is important to emphasize that the very name of "hyperbolic geometry" points on another way for the solution of Hilbert's Fourth Problem: searching for the new classes of "hyperbolic functions," which can be the basis for other hyperbolic geometries. Every new class of the hyperbolic functions generates its own new variant of the "hyperbolic geometry". By analogy with the "game of postulates" this way to solve Hilbert's Fourth Problem can be named the "game of functions" [19].

An original solution of Hilbert's Fourth Problem. For the first time, this way (the "game of functions") is used in the works $[7,8]$. The "game of functions" led in $[7,8]$ to the original solution of Hilbert's Fourth Problem. The essence of this solution consists in the following. Developing the idea of the metric form of Lobachevski's plane, known in hyperbolic geometry, the following formula for the metric form of Lobachevski's plane, based on the hyperbolic Fibonacci $\lambda$-functions (9), (10), has been derived in $[7,8]$ :

$$
(\mathrm{d} s)^{2}=\ln ^{2}\left(\Phi_{\lambda}\right)(\mathrm{d} u)^{2}+\frac{4+\lambda^{2}}{4}\left[s F_{\lambda}(u)\right]^{2}(\mathrm{~d} v)^{2}
$$

where $\Phi_{\lambda}=\frac{\lambda+\sqrt{4+\lambda^{2}}}{2}$ is the "metallic proportion" (13) and $s F_{\lambda}(u)$ is the hyperbolic Fibonacci $\lambda$-sine (9). The forms (18) are called the metric $\lambda$-forms of
Lobachevski's plane [7,8].

This formula gives an infinite number of new "Lobachevski's geometries" ("golden," "silver," "bronze," "copper" and so on to infinity) according to the used class of the hyperbolic Fibonacci $\lambda$-functions (9), (10).

The formula (18) sets an infinite number of the metric forms of Lobachevski's plane. This means that there is infinite number of Lobachevski's geometries, which are based on the "metallic proportions" (13). These new Lobachevski's geometries "with equal right, stand next to Euclidean geometry" (David Hilbert). Thus, the formula (18) can be considered as the partial original solution to Hilbert's Fourth Problem, based on the "game of functions". There is an infinite number of Lobachevski's geometries, described by the formula (18), which are close to Euclidean geometry. Every of these geometries manifest itself in Fibonacci $\lambda$-numbers (14), which can appear in physical world similarly to "Bodnar's hyperbolic geometry," where the classic Fibonacci numbers appear at the surface of phyllotaxis objects.

A new challenge to theoretical natural sciences. Thus, the main result of the research, described in [7-9], is a proof of the existence of an infinite number of the hyperbolic functions (9), (10), based on the "metallic proportions" (13). In addition, each class of the hyperbolic functions, corresponding to (9), (10), "generates" for the given $\lambda=1,2,3, \cdots$ its own "hyperbolic geometry," which leads to the appearance of the "physical world" with specific properties, which depend on the "metallic proportions" (13). The new geometric theory of phyllotaxis, created by Oleg Bodnar [4,5], is a striking example of this. Bodnar proved that "the world of phyllotaxis" is a specific "hyperbolic world," in which a "hyperbolicity" manifests itself in the "Fibonacci spirals" on the surface of "phyllotaxis objects".

However, the hyperbolic Fibonacci functions (4), (5), which underlie the "hyperbolic world of phyllotaxis," are a special case of the hyperbolic Fibonacci $\lambda$-functions (9), (10). In this regard, there are all reasons to suppose that other types of hyperbolic functions (9), (10), based on the "metallic proportions," can be good models for the new "hyperbolic worlds" that can really exist in Nature. Modern science cannot find these special "hyperbolic worlds," because hyperbolic functions (9), (10) were unknown until now. Basing on the success of "Bodnar's hyperbolic geometry" $[4,5]$, one can put forward in front to theoretical physics, chemistry, crystallography, botany, biology, and other branches of theoretical natural sciences the challenge to search the new "hyperbolic worlds of Nature," based on other classes of hyperbolic functions (9), (10).

In this case, perhaps, the next candidate for the new "hyperbolic world" of Nature (after "Bodnar's hyperbolic geometry") may be, for example, silver hyperbolic 
functions:

$$
\begin{aligned}
& s F_{2}(x)=\frac{\Phi_{2}^{x}-\Phi_{2}^{-x}}{\sqrt{8}}=\frac{1}{2 \sqrt{2}}\left[(1+\sqrt{2})^{x}-(1+\sqrt{2})^{-x}\right] \\
& c F_{2}(x)=\frac{\Phi_{2}^{x}+\Phi_{2}^{-x}}{\sqrt{8}}=\frac{1}{2 \sqrt{2}}\left[(1+\sqrt{2})^{x}+(1+\sqrt{2})^{-x}\right]
\end{aligned}
$$

which are connected with Pell numbers [12] and are based on the "silver proportion" $\Phi_{2}=1+\sqrt{2}$, connected with the fundamental mathematical constant $\sqrt{2}$.

\section{Back to Pythagoras, Plato and Euclid}

Platonic Solids and the golden ratio in the history of science. Differentiation of science and its division into separate spheres often do not allow to see a general picture of science and the main trends of its development. However, in science there are research objects, which unite disparate scientific facts into a coherent whole. Platonic solids and the golden ratio belong to the category of such geometric objects. The ancient Greeks raised them up to the level of the main indicators of the Universe Harmony, and put the problem of harmony in the center of their science.

Over the centuries, or even millennia, starting from Pythagoras, Plato, Euclid, these wonderful geometric objects have been the subject of admiration and worship of the brightest minds of mankind (Leonardo da Vinci, Luca Pacholi, Johannes Kepler in the Renaissance, Zeising, Lucas, Binet, Klein in the 19th century, Harold Coxeter, Nikolai Vorobyov, Verner Hoggat in the 20th century). The development of this direction has led to the creation of the "Mathematics of Harmony" as a new interdisciplinary direction of modern science. [9].

A role of the Platonic Solids and the Golden Ratio in modern science. The newest discoveries of modern science, based on the Platonic solids, the golden ratio, and the Fibonacci numbers, (fullerenes [20], quasicrystals [21], a new geometric theory of phyllotaxis [4,5], the "golden "genomatrices [22], the general theory of hyperbolic functions [7], the solution of Hilbert's Fourth Problem [7,8], algorithmic measurement theory and Fibonacci codes [23-25], the number systems with irrational base [26], the codes of the golden $p$-proportion [27], the "golden" number theory [28], the ternary mirror-symmetrical arithmetic [29], the generalized Fibonacci matrices [30], a new coding theory based on the Fibonacci matrices [31], "Proclus hypothesis" and the new view on the history of mathematics, starting from Euclid [32], the "golden" matrices [33], the Fibonacci Lorenz transformations and the "golden" interpretation of the special theory of relativity [34], the "Mathematics of Harmony" [9,35] and so on) create a general picture of the movement of modern science to the "Golden" Scientific Revolution what is one of the enduring trends in the development of modern science.

The most striking results of modern "mathematics of harmony" [9] are the following: the general theory of hyperbolic functions [7], which lead to the original solution of Hilbert's Fourth Problem [8] and new geometric theory of phyllotaxis ("Bodnar's hyperbolic geometry") $[4,5]$. "Bodnar's hyperbolic geometry" is the most convincing evidence of the numerical harmony of Nature. The above results relate to Lobachevski's hyperbolic geometry, which, according to academician Kolmogorov, is opening a new stage in mathematics development. Thanks to these scientific results, the hyperbolic geometry becomes a "harmonic theory". Its "hidden" harmony is expressed through the "golden ratio" and "metallic proportions".

\section{Conclusions}

1) The hyperbolic Fibonacci functions (2), (3) with the unique mathematical property (6) and the hyperbolic Fibonacci $\lambda$-functions (9), (10) with the unique mathematical property (17) are opening a new stage in the development of the hyperbolic geometry. The peculiarity of this stage consists in the fact that the creation of new hyperbolic geometries is based on the new classes of the harmonic hyperbolic functions (the "game of functions" [19]). If the hyperbolic Fibonacci functions (2), (3) were well-known to Lobachevski, the development of the hyperbolic geometry has gone in a new direction.

2) Bodnar's hyperbolic geometry, which is based on the Fibonacci hyperbolic functions (2), (3) and underlies the botanical phenomenon of phyllotaxis, is a brilliant example of the new class of hyperbolic geometry. The authority of Nature is hard to dispute. Bodnar's hyperbolic geometry is the most convincing proof of the uniqueness of the hyperbolic Fibonacci functions (2), (3). If we assume that Nature follows to the scenario, suggested by Oleg Bodnar [4,5], then we must conclude that Nature is a unique mathematician, who uses the hyperbolic Fibonacci functions (2), (3) for the creation of life forms.

3) The hyperbolic Fibonacci $\lambda$-functions (9), (10) generate an infinite number of hyperbolic geometries, which are close to Euclidean geometry. This fact is the basis for the original solution of Hilbert's Fourth Problem, which is described in [7,8]. The original solution of Hilbert's Fourth Problem puts forward in front of theoretical natural sciences a challenge to search the harmonic hyperbolic worlds, which can exist in Nature.

4) New approach to hyperbolic geometry is closely related to the Pythagorean doctrine of the numerical harmony of the Universe and Plato's cosmology, based on the Platonic Solids. Pythagoras and Plato's "harmonic ideas" were embodied in Euclid's Elements. The "harmonic ideas" by Pythagoras, Plato and Euclid are con- 
firmed brilliantly in modern science with the hyperbolic Fibonacci functions, "Bodnar's hyperbolic geometry" and the original solution of Hilbert's Fourth Problem.

\section{REFERENCES}

[1] A. P. Stakhov and I. S. Tkachenko, "Fibonacci Hyperbolic Trigonometry (Russian)," Reports of the Academy of Sciences of Ukraine, Vol. 208, No. 7, 1993, pp. 9-14.

[2] A. Stakhov and B. Rozin, "On a New Class of Hyperbolic Functions," Chaos, Solitons \& Fractals, Vol. 23, No. 2, 2004, pp. 379-389. http://dx.doi.org/10.1016/j.chaos.2004.04.022

[3] A. Stakhov and B. Rozin, "The Golden Section, Fibonacci series, and New Hyperbolic Models of Nature," Visual Mathematics, Vol. 8, No. 3, 2006. http://www.mi.sanu.ac.rs/vismath/stakhov/index.html

[4] O. Y. Bodnar, "The Golden Section and Non-Euclidean Geometry in Nature and Arts (in Russian)," Svit, Lvov, 1994.

[5] O. Y. Bodnar, "Dynamic Symmetry in Nature and Architecture," Visual Mathematics, Vol. 12, No. 4, 2010. http://www.mi.sanu.ac.rs/vismath/BOD2010/index.html

[6] A. P. Stakhov, "Gazale Formulas, a New Class of Hyperbolic Fibonacci and Lucas Functions and the Improved Method of the 'Golden' Cryptography," Academy of Trinitarism, Moscow. http://www.trinitas.ru/rus/doc/0232/004a/023210 63.htm

[7] A. P. Stakhov, "On the General Theory of Hyperbolic Functions Based on the Hyperbolic Fibonacci and Lucas Functions and on Hilbert's Fourth Problem," Visual Mathematics, Vol. 15, No. 1, 2013. http://www.mi.sanu.ac.rs/vismath/2013stakhov/hyp.pdf

[8] A. Stakhov and S. Aranson, "Hyperbolic Fibonacci and Lucas Functions, 'Golden' Fibonacci Goniometry, Bodnar's Geometry, and Hilbert's Fourth Problem," Applied Mathematics, Vol. 2, No. 3, 2011, pp. 283-293.

[9] A. P. Stakhov, "The Mathematics of Harmony. From Euclid to Contemporary Mathematics and Computer Science," World Scientific, London, 2009.

[10] V. G. Shervatov, "Hyperbolic Functions (in Russian)," Fizmatgiz, Moscow, 1958.

[11] V. de Spinadel, "The family of Metallic Means," Visual Mathematics, Vol. 1, No. 3, 1999. http://members.tripod.com/vismath/

[12] Pell Numbers. http://en.wikipedia.org/wiki/Pell_number

[13] M. J. Gazale, "Gnomon. From Pharaohs to Fractals," Princeton University Press, Princeton, 1999.

[14] Hilbert's Problems. http://en.wikipedia.org/wiki/Hilbert's_problems

[15] Hilbert's Fourth Problem. http://en.wikipedia.org/wiki/Hilbert's_fourth_problem

[16] D. Hilbert, "Mathematical Problems". http://aleph0.clarku.edu/ djoyce/hilbert/problems.html\#pr ob4

[17] A. V. Pogorelov, "Hilbert's Fourth Problem (in Rus-
sian),"Nauka, Moscow, 1974.

[18] B. H. Yandell, "The Honours Class-Hilbert's Problems and Their Solvers," A K Peters/CRC Press, Natick, 2003.

[19] A. P. Stakhov, "Non-Euclidean Geometries. From the 'Game of Postulates' to the 'Game of Function' (in Russian)," Academy of Trinitarizm, Moscow. http://www.trinitas.ru/rus/doc/0016/001d/00162125.htm

[20] Fullerene. http://en.wikipedia.org/wiki/Fullerene

[21] Quasicrystal. http://en.wikipedia.org/wiki/Quasicrystal

[22] S. Petoukhov, "Matrix Genetics, Algebra of the Genetic Code, Noise Immunity (in Russian)," Regular and Chaotic Dynamics, Moscow, 2008.

[23] A. P. Stakhov, "Introduction into Algorithmic Measurement Theory (in Russian)," Soviet Radio, Moscow, 1977.

[24] A. P. Stakhov, "Algorithmic Measurement Theory (News in Life, Science and Technology, Series "Mathematics and Cybernetics) (in Russian)," Knowledge, Moscow, 1979.

[25] A. P. Stakhov, "The Golden Section in the Measurement Theory," Computers \& Mathematics with Applications, 1989, Vol. 17, No. 4-6, pp. 613-638. http://dx.doi.org/10.1016/0898-1221(89)90252-6

[26] G. Bergman, "A Number System with an Irrational Base," Mathematics Magazine, Vol. 31, No. 2, 1957, pp. 98-119. http://dx.doi.org/10.2307/3029218

[27] A. P. Stakhov, "Codes of Golden Proportion (in Russian)," Radio and Communications, Moscow, 1984.

[28] A. P. Stakhov, "Generalized Golden Sections and a New Approach to Geometric Definition of a Number," Ukrainian Mathematical Journal, Vol. 56, No. 8, 2004, pp. 1143-1150. http://dx.doi.org/10.1007/s11253-005-0064-3

[29] A. P. Stakhov, "Brousentsov's Ternary Principle, Bergman's Number System and Ternary Mirror-Symmetrical Arithmetic," The Computer Journal, Vol. 45, No. 2, 2002, pp. 221-236. http://dx.doi.org/10.1093/comjnl/45.2.221

[30] A. P. Stakhov, "A Generalization of the Fibonacci Q-matrix," Reports of the National Academy of Sciences of Ukraine, Vol. 9, 1999, pp. 46-49.

[31] A. Stakhov "Fibonacci Matrices, a Generalization of the 'Cassini formula,' and a New Coding Theory," Chaos, Solitons \& Fractals, Vol. 30, No. 1, 2006, pp. 56-66. http://dx.doi.org/10.1016/j.chaos.2005.12.054

[32] A. P. Stakhov, "The Mathematics of Harmony: Clarifying the Origins and Development of Mathematics," Congressus Numerantium, Vol. 193, 2008, pp. 5-48.

[33] A. P. Stakhov, “The 'Golden' Matrices and a New Kind of Cryptography," Chaos, Solitons \& Fractals, Vol. 32, No. 3, 2007, pp. 1138-1146. http://dx.doi.org/10.1016/i.chaos.2006.03.069

[34] A. Stakhov and S. Aranson, "'Golden' Fibonacci Goniometry. Fibonacci-Lorentz Transformations, and Hilbert's Fourth Problem," Congressus Numerantium, Vol. 193, 2008, pp. 119-156.

[35] A. P. Stakhov, "The Golden Section and Modern Harmony Mathematics," Applications of Fibonacci Numbers, Vol. 7, 1998, pp. 393-399.. 